\title{
Philosophiques
}

\section{Modernité et utopie}

\section{Jean Roy}

Volume 6, numéro 1, avril 1979

URI : https://id.erudit.org/iderudit/203107ar

DOI : https://doi.org/10.7202/203107ar

Aller au sommaire du numéro

Éditeur(s)

Société de philosophie du Québec

ISSN

0316-2923 (imprimé)

1492-1391 (numérique)

Découvrir la revue

Citer cet article

Roy, J. (1979). Modernité et utopie. Philosophiques, 6(1), 3-44.

https://doi.org/10.7202/203107ar

Ce document est protégé par la loi sur le droit d'auteur. L'utilisation des services d'Érudit (y compris la reproduction) est assujettie à sa politique d'utilisation que vous pouvez consulter en ligne.

https://apropos.erudit.org/fr/usagers/politique-dutilisation/
Cet article est diffusé et préservé par Érudit.

Érudit est un consortium interuniversitaire sans but lucratif composé de l'Université de Montréal, l'Université Laval et l'Université du Québec à Montréal. Il a pour mission la promotion et la valorisation de la recherche. https://www.erudit.org/fr/ 


\title{
MODERNITÉ ET UTOPIE
}

\author{
par Jean Roy
}

\begin{abstract}
Dans ses grandes lignes, c'est le réalisme de Hegel qui s'est avéré propbétique, et ce sont les anticipations, prétendument progressistes, des utopistes (par exemple Platon, More, Campanella, Rousseau) qui se sont révélées, dans l'ensemble, régressives et archaïsantes.
\end{abstract}

À quarante ans, paraît-il, on sait que l'homme n'est pas heureux. "L'animal malade » n'arrive pas à se satisfaire de ce monde-ci. Il s'accommode mal, en effet, de la souffrance, de la maladie et de la mort, et ses peines lui paraissent plus vraies que ses rares moments de grâce. L'entendement philosophique a beau démontrer doctement qu'il s'agit là de phénomènes naturels liés à sa nature d'être fini, le cœur a ses raisons... Le brillant raisonnement d'Épicure - " tant que nous existons la mort n'est pas et quand la mort est là nous ne sommes plus ${ }^{1} "$ - n'entraîne pas une adhésion sans réserve. La force aveugle de la Nature, qui frappe les êtres que nous aimons et tend les pièges de notre défaite finale, nous laisse en proie à un sentiment de scandale aussi irrépressible qu'injustifiable.

Mais le sentiment de déréliction et de révolte contre la condition humaine n'est pas seulement cosmique, métaphysique, il s'insurge également contre la violence de l'histoire. Que la condition mortelle nous rassemble dans l'égalité commune devant le Destin: - " la mort n'est ni bourgeoise ni socialiste. Lorsque je parle de la mort, tout le monde me comprend ${ }^{2} \gg-$, cette pensée d'Ionesco peut nous consoler de notre impuissance, mais comment accepter le désordre et l'injustice du monde que nous créons ? L'homme fait l'histoire,

1. Épicure et les épicuriens, Textes choisis, PUF, p. 126.

2. G. IONESCO, Notes et contre-notes, NRF, 1962, p. 205. 
mais il ne fait pas celle qu'il veut. Dans cette éternelle ironie de l'Histoire se concentre notre illusion la plus tenace. "L'homme veut la concorde, mais la nature sait mieux que lui ce qui est bon pour son espèce : elle veut la discorde ${ }^{3}$ ", observe Kant. Certes, la ruse impersonnelle de la nature arrache les individus au mol oreiller de l'inertie et les pousse malgré eux, à la longue, vers un certain progrès. Mais les résultats sont-ils à la mesure des affres de la division ? Si seulement les hommes pouvaient se mettre d'accord une fois pour toutes sur un ordonnancement imparfait et au total tolérable de leur vie commune . . . Mais voilà, personne n'est disposé à supporter les frais de cette imperfection au profit d'autrui et il ne manque pas non plus de candidats pour tenir les meilleurs rôles. De plus, il existe des cœurs généreux qui s'indignent du sort immérité des uns et des autres. Avec la meilleure volonté, eux aussi aggravent le drame : "Dans tout homme sommeille un prophète, et quand il s'éveille il y a un peu plus de mal dans le monde...4 ». À la limite, en l'absence de graves injustices concrètes, la diversité des solutions envisagées pour fonder LA cité amicale et fraternelle de nos rêves suffit à attiser le feu de la violence. C'est ainsi que la seconde moitié du $\mathrm{XVI}^{\mathrm{e}}$ siècle européen est remplie de persécutions et de guerres dont les motifs nous apparaissent à peine intelligibles aujourd'hui. Au siècle suivant, Bossuet et Fénelon se querellaient encore sur la manière d'entendre le pur amour de Dieu ... La sagesse et le courage n'étaient-ils pas plutôt dans l'humour démobilisateur d'un Montaigne : "À la vérité, et je ne crains point de l'avouer, je porterais facilement au besoin une chandelle à S. Michel, l'autre à son serpent ${ }^{5}$."

Dans son étincelante démystification de l'optimisme leibnizien, Voltaire avait bien vu où faire porter la pointe de son attaque: le cours chaotique des affaires humaines. Au cours de leurs voyages, les globe-trotters de Candide parcourent la trame entière des malheurs naturels et de la folie des hommes: le recrutement forcé, la guerre, la vérole, la

3. E. KANT, "Idée d'une histoire universelle . . . ", in La philosopbie de l'bistoire, Aubier, 1947, p. 65.

4. E.M. CIORAN, Précis de décomposition, NRF, 1949, p. 11.

5. Montaigne, Les essais, III, chap. I, in Oetures completes, NRF, p. 769. 
tempête, le tremblement de terre, l'inquisition, la « rage des femmes ", la peste, les abus de pouvoir, l'oppression paternaliste, l'anthropophagie, l'esclavage, la fourberie, la rapacité, la piraterie, la vanité, l'orgueil nationaliste, la défiance, la prostitution, la satiété blasée, la superstition, l'exploitation du travail d'autrui, l'ennui, l'ambition. Seul le rêve de la société idéale, l'Eldorado, placé au centre de l'œuvre, distend momentanément la tresse de la violence de la nature et des passions humaines.

Schopenhauer a résumé la tragédie de la volonté dans son oscillation permanente entre la peine du travail et l'ennui engendré par l'oisiveté. La lutte contre la nécessité n'a pas aussitôt commencé à goûter sa victoire que l'énergie vitale inemployée piétine dans l'ennui. Alors, les hommes cherchent avidement quelque aventure, même fort exigeante, qui les remette en mouvement. La tranquillité menacée, les chefs politiques habiles réorientent l'enthousiasme sur des voies d'évitement : la satisfaction des besoins vitaux et des jeux innocents - Panem et circenses ! Le danger subsiste malgré tout, malgré l'espèce de compromis tacite qui règle la vie des sociétés : «Comme le besoin pour le peuple, l'ennui est le tourment des classes supérieures. Il a dans la vie sociale sa représentation le dimanche; et le besoin, les six jours de la semaine $^{6}$. »

L'imagination utopique tire précisément son énergie de la conviction que cette situation n'est pas fatale : un monde autre est possible où seraient surmontées les vicissitudes du monde présent. La pérennité du malheur passé ne préjuge aucunement de l'avenir. La dialectique de l'utopie écrite ou pratiquée implique une prise de conscience critique de l'actuel immédiat éprouvé comme intolérable et une construction souvent très détaillée d'une société conforme aux exigences d'un nouvel

6. A. Schopenhauer, Le monde comme volonté et comme représentation, PUF, 1966, p. 396. Ce jugement, valable pour la société traditionnelle, demanderait sans doute à être nuancé pour la société technicienne ainsi que le suggère T. Parsons : « Les groupes professionnels supérieurs de la société moderne, loin de constituer une "classe de loisir " font généralement partie des groupes qui dans l'histoire de l'humanité, travaillent le plus intensément. Paradoxalement, la classe laborieuse prétendument exploitée s'est bien plus rapprochée de la "classe de loisir " dans la société moderne. "Le système des sociétés modernes, Dunod, 1973 , p. 119. 
idéal. Ce rythme d'arrachement et de projection est clairement marqué dans la structure même de l'œuvre tenue pour le paradigme du genre, L'utopie de Thomas More. Le premier livre, en effet, critique les tares de l'Angleterre de l'époque: l'ambition des puissants, la flagornerie des conseillers clairvoyants mais lâches et le silence intéressé des incapables, les peines excessives et d'ailleurs inefficaces contre la multiplication des atteintes à la propriété, le trop grand nombre des nobles, véritables parasites collés au flanc de la classe productive, la convoitise des riches, voire "même de très saints abbés ", la multiplication désordonnée des pâturages et l'accroissement des chômeurs acculés au vol, au vagabondage, à la mendicité, la cherté des vivres, la prostitution, l'ivrognerie, " l'abandon de millions d'enfants aux ravages d'une éducation vicieuse et immorale ", l'incurie de la société vis-à-vis de ses malades et de ses vieillards, etc. Le second livre décrit avec précision les institutions et les mœurs des Utopiens. Les deux livres composent donc un diptyque parfaitement contrasté et définissent le mouvement intérieur de l'œuvre.

Le fait est significatif: l'épicentre de la plus grande densité d'émissions utopiques procède régulièrement des périodes de crise et des groupes traqués par l'histoire. Dans le sentiment de détresse qui étreint les hommes de ce temps éclaté, la conscience du mal rebondit en aspiration délirante, à la mesure de la profondeur vécue du désordre. Plus l'impuissance réelle est sans espoir immédiat, plus l'impatience se précipite, en imagination, vers des images d'accomplissement total comme si à l'absence des moyens une réalisation fictive des fins apportait une nécessaire compensation et le principe du salut. Dans l'effondrement du sacré traditionnel des valeurs et de proche en proche de la hiérarchie organisatrice de l'ordre social, l'imagination utopique avance aussitôt un monde autre, une alternative, une nouvelle échelle sacralisée des préférences du groupe, c'est-à-dire selon l'étymologie du mot une nouvelle biér-archie. Cet exercice mental, apparemment gratuit et pour ainsi dire ludique, procède en réalité du sentiment d'avoir à répondre à une tâche urgente, indéclinable. Nulle société ne peut durer sans un minimum de cohésion morale. Assailli par les convulsions d'une insupportable histoire, l'utopiste riposte 
par la création d'une espérance qui apporte un sens neuf pour la vie et l'action de tous.

Or, contrairement à l'opinion courante, on peut douter des vertus prophétiques de l'utopie: si l'on confronte, en effet, ses anticipations à l'effectivité du processus historique, on doit plutôt convenir de la pauvreté de ses facultés visionnaires. Bien plus, dans les recoupements de la projection prétendument progressiste et de la réalité, ce que l'histoire ultérieure a incarné dans la douleur répète trop souvent sur une autre échelle, sous des couleurs différentes, les figures les plus hideuses du passé. Summum jus summa injuria, dit le proverbe latin. De la même manière, ne pouvons-nous pas dire à notre tour que le désir perfectionniste ${ }^{7}$ d'une société parfaitement intégrée, unanime, ne peut être fugitivement approché que par des moyens violents et contraires à l'humanité ? Paradoxalement, l'homme des temps nouveaux regarde vers un avenir radieux comme dans un miroir tourné vers le passé. Invariablement, la réfection démiurgique du monde revient à la petite communauté close, à la chaleur humaine des origines de l'espèce. S'il faut chercher un répondant historique au rêve rigoureux de l'utopie, c'est vers les sectes, communautés, communes, ordres monastiques et militaires, tantôt attentistes, tantôt activistes qu'il faut se diriger. Cette hantise de la communauté primitive étendue aux grands espaces, aux grands nombres et prétendant à la synthèse définitive aboutit, au XXe siècle, aux totalitarismes de gauche ou de droite, belliqueux ou repliés sur eux-mêmes.

Dressée contre le carnage de l'histoire, la révolte utopique entretient des rapports essentiellement contradictoires au temps : elle opère à contre-temps, comme par définition. C'est ainsi que les temps modernes, période d'intense « accélération de l'histoire », soulèvent un riche parallèle de reconstructions imaginaires et fournissent un champ d'études privilégié au chassé-croisé des deux plans. Qu’il s'agisse de la tradition dominante, ascétique et autoritaire jusquà la manie, ou de la tradition hédoniste et libertaire (Rabelais, Fourier, Marcuse),

7. On Lira avec profit le chap. IV du livre de G. SARTORI, Democratic Theory, Praeger, 1965, "Perfectionism and Utopia". 
l'utopie manque l'essence de la société moderne ou plutôt de la modernisation occidentale. Tantôt passéiste, tantôt futuriste, elle demeure en deçà ou se jette d'emblée au-delà de la modernité, encore que le rapport schizophrénique au présent ne soit pas sans failles. Du reste la modernité n'est pas une substance ou une chose parfaitement délimitable, mais un processus aux contours mouvants selon la diversité des orientations sociologiques.

Il n'est pas question dans le cadre de ce texte d'exposer une théorie complète de la modernité ; nous nous contenterons d'en indiquer les éléments les plus saillants pour les besoins de notre propos. Dans cette optique, la politique hégélienne nous parât se distinguer par son exceptionnelle richesse. D'autre part, la littérature utopique appelle un choix fort limité. Pour la commodité de l'exposé, nous ne retiendrons que les noms de Platon, More, Campanella et Rousseau dont nous ne soulignerons que certains aspects destinés à vrai dire à illustrer notre thèse plutôt qu'à la démontrer dans toute son ampleur.

Ce n'est pas par hasard que Hegel, en qui Talcott Parsons voit l'auteur "d'une théorie raffinée de l'évolution sociale générale et de son apogée dans l'Occident moderne ${ }^{8}$ ", se voit rangé par Mannheim dans le camp le plus opposé à l'utopie telle qu'il la définit. En effet, si l'utopie exprime selon lui la tension révolutionnaire vers un avenir en totale rupture avec l'enfer du présent, la pensée de Hegel évolue en sens inverse en déployant la rationalité profonde du processus réel de l'Esprit. L'actualité n'est plus éprouvée comme le lieu du mal, mais réfléchie comme l'incarnation vivante de la marche concrète de l'Idée. Au sentiment hargneux du moraliste, à l'émigration abstraite dans le monde creux du rêve, Hegel oppose le cours intelligible de l'Histoire et la réconciliation consciente avec la Sittlichkeit. Le hic et nunc n'est plus nié comme l'autre de l'exigence et de l'espérance, mais comme le support certes imparfait mais réel de la moralité et de l'accomplissement humain total. Sans aller jusqu'à voir dans Hegel « le penseur prototypique de la gauche ${ }^{9}$ ", on ne peut le placer non plus

8. T. PARSONS, op. cit., p. 1.

9. T. Molnar, La gaucbe vue d'en face, Seuil, 1970, p. 33. 
dans le camp des conservateurs ainsi que le fait Mannheim ${ }^{10}$. À vrai dire, Hegel répudie à la fois un idéalisme quiétiste ou activiste et un positivisme négateur de toute transcendance; sa politique se tient à égale distance de la sacralisation apologétique du statu quo et de l'idée intemporelle du platonisme qui sont deux manières d'évacuer la vérité concrète du devenir. L'idée hégélienne de l'État moderne n'exclut pas tout aspect normatif : elle écarte seulement un Sollen vague et abstrait, qui flotte au-dessus de l'empirie, et non pas la rationalité immanente de l'évolution sociale. B. Quelquejeu définit correctement le statut théorique des Principes de la pbilosopbie du droit lorsqu'il écrit : "L'État hégélien n'est ni un idéal conçu, ni une réalité observée ; il est le déchiffrement en profondeur d'une puissance sensée à l'œuvre dans l'histoire, (. . .) il est un sens dynamique, une finalité interne qui s'effectue. En déclarant que l'État est l'effectivité de l'idée éthique, de la liberté concrète et de la volonté substantielle, Hegel formule une proposition affirmative qui n'est ni un présent descriptif, ni un présent optatif ou utopique, mais ce qu'on pourrait appeler un présent essentiel : celui d'un sens en marche, à l'œuvre dans l'histoire, et que la pensée peut atteindre et saisir, celui d'une essence s'effectuant historiquement et réellement dont la philosophie peut et doit identifier la constitution ${ }^{11}$ ".

Or, comment Hegel définit-il le mouvement global de la modernisation? C'est dans le fameux paragraphe 260 qu'il nous en donne peut-être la formulation la plus concise : " Le principe des États modernes a cette puissance et cette profondeur extrêmes de laisser le principe de la subjectivité s'accomplir jusqu’à l'extrémité de la particularité personnelle

10. K. Mannheim, Idéologie et utopie, Rivière, 1956, p. 181-196. Du reste, cette assignation ne va pas sans forcer Mannheim à réviser sa distinction fondamentale. Après avoir annoncé le conservatisme dans les configurations modernes de la mentalité utopique, il rectifie : «La mentalité conservatrice comme telle n'a pas d'utopie. Idéalement, elle est dans sa structure mêrme, complètement en harmonie avec la réalité dont elle a, pour le moment, conquis la mâttrise " (p. 182). Sous le fouet de l'attaque contre-idéologique, il ne reste plus à I'affirmation conservatrice qu'à se muer en contre-utopie consciente.

11. B. Quelquejeu, La volonté dans la philosophie de Hegel, Seuil, 1972, p. 308-9. Eric Weil exprimait déjà la même idée à sa manière : "Cette "idée " (de l'État) est donc normative dans ce sens qu'elle donne la possibilité d'apprécier ce qui existe. Mais dans un autre sens elle n'est pas normative (et ce point est décisif) : elle ne donne pas un sens intemporel ou extra-temporel. "Hegel et l'État, Vrin, 1950, 2e édition, 1966, p. 28, note 12 . 
autonome et en même temps de le ramener à l'unité substantielle et ainsi de maintenir cette unité dans ce principe lui-même. "Ce mouvement d'intégration différenciée se réfléchit dans la structure même de l'œuvre. Le "droit abstrait" et «la moralité » affirment des principes radicalement individualisants : le principe romain-bourgeois de l'individu comme sujet de droit et le principe chrétien-kantien de la subjectivité infinie et autonome. Tous deux ont disloqué l'univers spirituel de la belle Totalité grecque. La morale vivante, l'éthos socio-politique lié à la triple institution de la famille, de la société civile et de l'État, fournit un contrepoids objectif et public au mouvement de privatisation de la première et de la deuxième partie. Le propos est donc clair : il s'agit de ré-inscrire dans la pensée politique le sens grec de la totalité cohérente après des siècles de civilisation chrétienne et l'avènement de la société bourgeoise. Par ce biais, il est vrai, Hegel communique avec la hantise utopique et romantique de la communauté homogène. Mais s'il est demeuré nostalgique de la synthèse organique de la Polis, après la "transvaluation " chrétienne des valeurs comme après la révolution de 89 et la révolution industrielle en plein essor (dont il mesure d'emblée l'extrême portée historique), il est vain de rêver à la réactivation immédiate des valeurs perdues. Cette Europe qui l'ennuie $^{12}$ a vivement besoin d'un nouvel esprit civique. Mais il n'est pas question de tourner le dos au dynamisme de l'histoire. La nouvelle synthèse politique ne peut restaurer la prémodernité, mais doit assumer les forces vives du présent et sa rationalité tendancielle. Selon l'exigence du dépassement dialectique, l'organisation politique moderne doit retenir en elle les différences aiguisées par le devenir: tout autre prétendu "dépassement " ne pourrait être qu'évasion abstraite, régressive, appauvrissante. De ce point de vue, on a du mal à comprendre comment on a pu compromettre Hegel avec les totalitarismes et les régimes révolutionnaires : l'effectuation concrète de la liberté raisonnable implique la médiation de la communauté familiale et de la société civile à l'intérieur de

12. J. D'HONDT rapporte qu'il citait avec complaisance l'exclamarion désabusée de Napoléon : "cette vieille Europe m'ennuie !" Déjà il pressentait que “ si l'on veut décamper l'Europe (über Europa hinausschicken), ce ne peut être que du côté de l'Amérique ". Hegel, Philosophie de l'bistoire vivante, PUF, 1966, p. 25. 
laquelle les corporations jouent le rôle de seconde famille. Ces institutions ne sont pas simplement les organes de transmission du commandement politique mais jouissent, comme dans tout régime libéral, d'une autonomie et d'un pouvoir de pression relatifs. L'affirmation de la prééminence de «l'État politique " comme but en soi (selbstzweck) n'implique pas l'absorption des instances institutionnelles inférieures. De plus, la plupart des libertés individuelles que nous connaissons dans les démocraties occidentales sont formellement consacrées dans les Principes. S'il se montre méfiant à l'égard du $\delta \epsilon \mu o s$ et de « la masse informe », nous aurions pourtant tort de voir en lui un adversaire du libéralisme politique ${ }^{13}$.

L'inspiration qui commande la Sittlichkeit rompt avec l'individualisme du libéralisme classique auquel le nom de Locke est attaché. En effet, on ne procède pas ici à partir de la multiplicité abstraite d'individus adultes, "sortis de terre comme des champignons" selon la fiction de Hobbes, en quête de la sécurité de leurs droits, mais bien plutôt, comme chez Platon et Aristote, à partir des communautés concrètes à l'intérieur desquelles l'individu se développe et cherche "le bien vivre ". Mais le traitement hégélien du contenu se réfere avec réalisme à la condition des hommes de son temps qui, dans ses grandes lignes, demeure encore celle de notre actualité.

En un sens, l'union consentie de l'homme et de la femme dans le mariage représente le type de la reconnaissance réciproque que la Phénoménologie avait défini comme "un Je qui est un Nous et un Nous qui est un Je ". Sur ce point, le jeune Marx posera ses pas dans ceux de Hegel : "Le rapport immédiat, naturel, nécessaire de l'homme à l'homme est le rapport de l'homme à la femme ${ }^{14}$." Certes le mariage est

13. La confusion courante à ce sujet tient au fait que les idéaux de la démocratie et de la liberté sont confondus sur la base de l'expérience des démocraries libérales d'Occident tenues pour normales et pour canoniques. Or, c'est la synthèse démocratico-libérale qui demande une explication et la "démocratie totalitaire" qui s'inscrit dans la pente naturelle du Pouvoir. Pour des éclaircissements conceptuels sur cette distinction, on peut se reporter à l'ouvrage déjà cité de G. SARTORI ; pour l'aspect historique de la question, on lira avec profit J.L. Talmon, Les origines de la démocratie totalitaire, Calmann-Lévy, 1966.

14. K. MARX, Les manuscrits de 1844, Éditions sociales, 1962, p. 86. 
inauguré par un contrat et les conséquences juridiques en apparaissent pleinement au moment de sa dissolution, mais la plénitude concrète de la vie du couple dépasse l'aspect d'un arrangement intéressé entre personnes autonomes usant mutuellement de l'autre comme d'un moyen. Bien que le lien familial se situe très loin, après la dialectique des volontés, par-delà les médiations du droit abstrait et de la moralité, en dehors de l'ordre extérieur des intérêts particuliers, il réalise la destination communautaire de la vie politique " dans sa phase naturelle ou immédiate ${ }^{15}$ ". Par-delà l'ordre atomisé et inconscient de «la main invisible" qui gouverne la société, l'universel concret de l'État reprend le motif totalisant de la famille, mais dépouillé de l'étroitesse de sa particularité et de son enracinement dans l'immédiateté de la vie : la synthèse est alors consciente et volontaire, médiatisée et universelle. La vie éthique trouve son achèvement dans "l'altruisme universel » de l'État après avoir traversé "l'altruisme particulier " de la famille et "l'égoïsme universel " de la société civile ${ }^{16}$. Mais ni la société ni l'État ne peuvent être pensés comme de simples prolongements de la famille. Tout au contraire, Hegel accuse le contraste croissant entre l'intimité sentie de la Gemeinschaft familiale et l'artificialité impersonnelle de la bürgeliche Gesellschaft. Avec un réalisme aigu, il analyse la novation essentielle : la rationalisation rapide de l'activité économique qui cesse de se développer dans le cadre restreint de la famille, cependant que la société et l'État assument de plus en plus des charges qui lui étaient autrefois dévolues (éducation, bienfaisance privée et assistance publique, tutelle en certains cas, etc.). "Ainsi l'individu est devenu fils de la société civile qui a envers lui autant d'exigences qu'il a de droits sur elle ${ }^{17}$. »

Certes, la rupture n'est pas totale: le travail et la satisfaction des besoins sont la grande affaire de la société. Or, comme l'a magistralement montré $\mathrm{H}$. Arendt, le travail et la consommation ne sont que les deux moments du processus

15. HeGel, Principes de la philosophie du droit, trad. A. Kaan, NRF, 1940, \#\# 33, 157. G. Fessard va jusquà ècrire que "le principe familial est à l'origine de la société civile et de l'État ". "Les relations familiales dans la philosophie du droit de Hegel, "Hegel-Jahrbuch, 1967, p. 47.

16. Cf. Schlomo Avineri, Hegel's Theory of the Modern State, Cambridge, 1972, p. 134.

17. Hegel, Principes. . ., \# 238. 
cyclique de la vie. De ce point de vue, la société moderne peut être comprise comme un vaste ménage collectif. Toute la société est mobilisée prioritairement dans le renouvellement incessant du processus vital. Sous le couvert de "l'humanisme du travail ", l'organisation technique de la production a, en fait, refoulé la morale aristocratique et héroïque du maître et adopté les valeurs de l'esclave ${ }^{18}$.

Mais ce changement d'échelle et ce renversement de valeurs sont solidaires d'une certaine autonomisation de l'ordre économique. De même que Galilée et Machiavel ont distendu les liens traditionnels qui unissaient la science et la politique à la religion et à la morale, de même les économistes anglais ont dissocié l'économie de ses vieilles solidarités. La société industrielle ne connaît qu'un sacré, celui de l'efficacité de ses opérations: elle s'efforce de satisfaire les besoins qui se manifestent sans prétendre les juger ni tenter de choisir entre eux. D'autre part, indifférente à l'individu et à ses qualités elle s'accommode volontiers d'un grand nombre d' " hommes sans qualités " - elle ne connaît que des rôles et des fonctions dans le système récurrent production-consommation. On le voit, son caractère propre est la neutralité morale. Bien plus, ainsi que le montrera Mandeville avec une pointe de provocation: "les vices privés profitent au public". Rendue à la pureté de son essence, l'économie n'a cure des mobiles égoïstes de l'individu, pourvu que son dynamisme concoure à la prospérité générale. Délié de ses attaches, le $\mu \epsilon ́ \gamma \alpha \rho o \nu$ social est le lieu reconnu de la libre concurrence des intérêts particuliers : chacun y est traité en tant que moyen éventuel de la satisfaction propre. Les fins de l'individu sont médiatisées par les besoins des autres; l'individu actif et clairvoyant capable de répondre à leurs demandes maximisera du même coup ses avantages. L'intérêt particulier d'un individu ou d'une corporation n'est pas l'objet d'une condamnation dans son principe, mais seulement dans ses effets possiblement préjudiciables.

Bien plutôt, par son principe même la société bourgeoise stimule "le mauvais infini du désir ". L'incroyable avidité du consommateur n'est pas une conséquence accidentelle du

18. Hannah ARENDT, Condition de l'homme moderne, Calmann-Lévy, 1961. 
Système, mais se trouve inscrite dans sa logique même ${ }^{19}$. L'insatisfaction revendicative est l'atmosphère propre d'un tel type de société. L'enrichissement de l'ensemble, bien qu'inégal selon les "états" et les classes, et l'anticipation subjective de l'amélioration du sort personnel avivent sans cesse cette frustration. Cependant, à l'intérieur de la nation c'est moins l'élévation globale du niveau de vie et la victoire sur la famine ${ }^{20}$ qui sont ressenties que les inégalités dans la répartition de la richesse produite en commun à travers une division croissante des tâches. C'est pourquoi la diffusion du thème de l'exploitation croissante au moment où elle cessait d'exprimer la réalité sociale en cours de transformation n'est qu'un paradoxe apparent. Dans une société de petits propriétaires (paysans et artisans), relativement indépendants les uns des autres, chacun n'est redevable du fruit de son travail qu'à lui-même... et aux aléas de la température. Mais dans le cadre moderne de la division du travail, la contribution de chacun à la richesse générale ne peut être qu'appréciée diversement. Encore une fois Hegel relève avec perspicacité l'essentielle dimension psychologique de la classe sociale et notamment de la plèbe (Pöbel) :

« Si une grande masse descend du minimum de subsistance qui apparaît de soi-même comme régulièrement nécessaire pour un membre d'une société, si elle perd ainsi le sentiment du droit, de la légitimité et de l'honneur d'exister par sa propre activité et son propre travail, on assiste à la formation d'une plèbe qui entraîne en même temps avec soi une plus grande facilité de concentrer en peu de mains des richesses disproportionnées ${ }^{21}$. »

19. J. FouRAstié l'observe justement : « Les besoins, loin de s'apaiser lorsque le niveau de vie s'élève, se développent au contraire. Aucune limite, aucune lassitude de l'appétit de consommation ne s'est jamais laissée déceler sur les statistiques, quelle que soit l'importance des revenus dépensés. " Les 40000 beures, Laffont-Gonthier, 1965, p. 38-39.

20. FOURASTIÉ situe symboliquement l'avènement de la société de consommation en 1709 , au moment de la dernière grande famine française. Cf. L'Express, 9-15 février 1970, p. 70-71.

21. Hegel, Principes ... \# 244. La remarque de Hegel à l'égard de ce paragraphe est éloquente: "Le plus bas niveau de subsistance, celui de la masse pauvre, est fixé automatiquement mais le minimum en varie considérablement d'un pays à l'autre. En Angleterre, même les plus pauvres croient qu'ils ont des droits. (. . .) Contre la nature l'homme ne peut élever la prétention d'aucun droit, mais dans une société établie la pauvreté prend immédiatement la forme d'un tort fait à une classe par une autre. L'importante question des moyens d'abolition de la pauvreté est l'un des problèmes les plus troublants qui agitent la société moderne. " 
Ainsi dans le contexte de l'économie libérale moderne, les progrès du machinisme engendrent à la fois la richesse et la conscience blessée de la pauvreté comme d'un tort fait à une classe par une autre, une socialisation poussée et le sentiment de l'isolement, de l'insécurité, de l'impuissance, bref le sentiment de l'aliénation.

Le problème de l'intégration du Pöbel dans l'État moderne, Hegel l'avoue, n'est pas résolu. Certes, il envisage certains moyens de correction : l'action volontaire d'institutions caritatives privées, la redistribution de la richesse par la taxation directe, la mise en place de travaux publics, le développement de marchés extérieurs, le colonialisme, etc. Toutefois, aucun de ces éléments ne constitue à ses yeux une vraie solution dans le cadre de sa philosophie. En effet, d'un côté, l'État ne peut rester totalement à l'écart de la compétition économique sous peine d'exclure toute une catégorie de citoyens de la participation effective à sa tâche rationnelle et raisonnable: Hegel conclut donc à la nécessité d'un certain interventionnisme étatique. Mais d'un autre côté, si l'État assume le contrôle total et direct de la collectivité, il en résulte une synthèse archaïque, tel «le despotisme oriental» de l'Égypte pharaonique, c'est-à-dire une intégration forcée et indifférenciée 22 .

Dans l'atomisme de la société civile, la corporation est appelée à jouer un rôle capital en vue de la réconciliation voulue et médiatisée de la particularité et de l'Universel, de la liberté et de l'organisation, du sentiment communautaire et de la rationalité instrumentale du Not - und Verstandesstaat ${ }^{23}$. Hegel ne jette aucun discrédit sur l'égoösme individuel ou corporatif : vouloir anéantir la force de ces appétits est une entreprise vouée à l'échec, utopique. Mieux vaut les canaliser à l'intérieur d'institutions qui les dépouillent de leur sauvagerie naturelle et en recueillent le dynamisme sous l'arbitrage supérieur de « l'État politique ». La reconnaissance légale de la corporation légitime formellement ce principe de l'intérêt particulier. Dans un monde fondé sur la compétition, la corporation, seconde famille ${ }^{24}$, non seulement joue une fonc-

\footnotetext{
22. lbid., \# 236 .

23. Ibid., \# 183 .

24. Ibid., \# 255 .
} 
tion de protection économique, mais aussi recompose un tissu organique de solidarité vécue. Cependant, elle n'est pas une caste fermée ; ouverte, elle permet la mobilité sociale. D'autre part, en l'absence des partis politiques que nous connaissons aujourd'hui ${ }^{25}$, l'État y trouve une base de représentation. En effet, les députés de la Chambre basse sont élus par les corporations et les communautés diverses ${ }^{26}$. Quant à l'individu, sa participation à la vie de son groupe professionnel le prépare et l'éduque aux rôles directement politiques.

On le voit, Hegel est allé droit à l'essentiel : les relations sociales dans la ville moderne sont de plus en plus médiatisées par des objets et par l'objectivation fonctionnelle. Corrélativement, la subjectivité se trouve à la fois plus libre et plus dépendante : mieux organisée, la société décharge l'individu de tâches qui étaient autrefois les siennes, mais en même temps il est happé dans un réseau complexe d'activités formant un tout rationnel, un système d'interdépendances universelles. Le prix à payer est assurément très élevé. Outre le fait que la société ne parvient pas à résorber par elle-même la pauvreté d'une partie importante de la collectivité, la division technique et sociale du travail ("le travail en miettes") détruit le rapport immédiat et vivant de l'homme à ses ouvres ainsi que les relations "organiques" des hommes entre eux. Enfin, elle ne véhicule aucun sens. Comme le diront Weber et Habermas, elle est le lieu d'actions rationnelles par rapport à une fin techniquement définie comme objectif (Zweckrational), et non pas sensées par rapport à une valeur inépuisable (Wertrational).

Que faire ? Hegel récuse autant les réactionnaires, nostalgiques du "bon vieux temps ", que les révoltés en proie à un activisme abstrait ou, pour le dire autrement, les traditiona-

25. Les partis, il ne faut pas l'oublier, sont un phénomène récent. “En 1761 (en Grande-Bretagne), nous tappelle Sir Lewis Namier, aucune élection au Parlement n était le fait des partis; en 1951 pas un seul candidat indépendant n'a été élu. "Non seulement les partis n'ont guère d'influence avant le deuxième quart du XIXe siècie, mais ils n'existent pas ou, si l'on préfere, ce qui en tient lieu, la réalité que recouvre le mot de "parti " n'a que de très lointains rapports avec les partis tels que nous les connaissons maintenant". J. CHARlot, Les partis politiques, A. Colin, 1971, p. 4.

26. Hegel, Principes... \#\# 308, 311. 
listes et les sentimentaux ${ }^{27}$. Qu'ils rêvent d'impossibles restaurations ou qu'ils se précipitent d'un bond à « la fin de l'histoire », ils tentent vainement de s'évader de l'historicité concrète. Il n'est pas hostile aux novations; encore faut-il qu'elles s'articulent sur des forces réelles et qu'elles accomplissent la rationalité tendancielle du devenir. S'il ne s'agit que de se complaire dans le sentiment de sa noblesse morale sur la base d'intentions généreuses, l'utopie de la fraternité universelle peut être d'un grand secours. Mais s'il s'agit d'œuvrer à la paix entre ennemis, la pression de l'utopie sur l'action politique risque d'être catastrophique si elle n'est pas contenue par les médiations élémentaires de la concorde appuyées en dernière instance sur la contrainte légitime. G. Fessard observe le total silence de la Pbilosophie du droit sur la notion de fraternité en tant que fondement possible de la société et de l'État ${ }^{28}$. S'il faut chercher une trace de cette valeur, c'est dans le sentiment patriotique qu'il faut en voir la reprise partielle, mais il ne saurait être confondu avec le nationalisme chauvin et agressif. Quant à la fraternité révolutionnaire, nul mieux que lui n'a saisi le lien intime qui la relie à la Terreur. Si Hegel dépasse la téléologie classique ou politique, comme agent de paix et de sécurité des droits individuels, et vise la réaffirmation d'une authentique communauté politique, il échappe pourtant aux séductions eschatologiques du révolutionnarisme abstrait.

Sartre qui la « répète » en inverse totalement la pointe. Au lieu de mettre en question la pureté de l'idéal à partir de ses conséquences terroristes, il exalte la violence épuratrice pourvu qu'elle invoque la Fraternité. Il préfere l'immédiateté abstraite des lynchages perpétrés par le groupe en fusion aux médiations juridiques du groupe assermenté, structuré, organisé, différencié. La suspicion maladive à l'égard de toute différence et finalement à l'égard de l'altérité elle-même, encouragée par le vague idéologique ou plutôt "utopique » de la Fraternité, laisse la voie libre à l'arbitraire du Souverain.

27. J. D'Hond, "L'histoire et les utopistes selon Hegel et Marx ", in De Hegel à Marx, P.U.F., 1972 ; Éric WEIL, "La morale de Hegel " in Essais et conférences, t. I, Plon, 1970 , p. $142-158$. 
Loin d'aménager institutionnellement des libertés limitées mais réelles, Sartre identifie le pratico-inerte avec la négation de la praxis révolutionnaire, elle-même assimilée à la plénitude de la liberté collective. "La liberté du vide ", "les abstractions sans Idée » que Hegel dénonçait naguère conservent à n'en pas douter un indéracinable attrait.

L'État moderne est l'État d'une société moderne, mais, en tant qu'État, il transcende le plan purement social des intérêts particuliers. La taxation et la guerre font éclater le principe utilitaire de l'intérêt bien compris qui règle le mouvement intérieur de la société et qui pèse abusivement sur les représentations dices du droit naturel moderne quant au fondement du politique.

Ce qui frappe d'abord, c'est la croissance ininterrompue d'un État centralisé dont la France et la Prusse ont fourni des modèles particulièrement éclatants. De l'Ancien Régime à la période postrévolutionnaire, Tocqueville comme Marx s'accordent sur la nature du phénomène, soit pour s'en inquiéter, soit pour en prédire le dépérissement final à la suite d'une exacerbation révolutionnaire des contradictions sociales.

Ce gonflement de la sphère étatique ne va pas sans un important développement bureaucratique. Hegel voit dans cet État reposant sur un fonctionnarisme ouvert, accessible aux talents sélectionnés par voie de concours et imbu du sens de l'État, un contrepoids universalisant par rapport à la dispersion et aux conflits des intérêts particuliers (bien qu'il observe avec réalisme que la plupart proviennent de la classe moyenne). De la même manière, la classe bureaucratique incarne la fonction rationnelle et critique en face de la fonction vitale de la paysannerie et du conversatisme de la noblesse terrienne. Si le mot bureaucratie éveille des résonances déplaisantes à nos oreilles, il n'en reste pas moins que dans le contexte de la Prusse de l'époque, elle représentait un facteur de progrès : «Elle est alors pratiquement la seule force politique qui résiste aux féodaux, écrit J.D'Hondt. (. . .) Les rares révolutionnaires prussiens du XVIII ${ }^{\mathrm{e}}$ siècle et du début du XIX ${ }^{\mathrm{e}}$ siècle sont sortis de leurs rangs - par exemple les dirigeants de la 
conspiration des Évergètes, certainement bien connue de Hegel $^{29} »$.

Mais surtout, l'État s'est séparé de l'Église et se veut laï, séculier, dégagé de toute prétention à la domination des esprits. La constitution de l'État moderne innove à cet égard. Bien que Hegel n'entende pas la notion de constitution dans son sens strictement juridique ou dans le sens idéologique de la tradition libérale (Locke, Montesquieu, Kant, Constant, Stuart Mill), mais en premier lieu dans le sens «organique » qu'il revêt dans la politique classique ${ }^{30}$, il ne récuse pas non plus l'apport de la codification écrite, systématique ${ }^{31}$ et le sens des libertés individuelles véhiculé par le constitutionnalisme. De ce point de vue, l'absolutisme monarchique et le démocratisme tyrannique de la seule majorité manquent également le sens à la fois concret et raisonnable de la constitution : il ne suffit pas de rédiger une constitution pour modeler effectivement un peuple ; à proprement parler on ne fait pas une constitution, « chaque peuple a la constitution qui lui convient ${ }^{32} »$. Encore ici, Hegel procède en sens inverse du style aprioristique et déductif des faiseurs d'utopies. D'autre part, c'est le propre d'un État despotique de n'accepter aucune limitation légale à l'arbitraire total de son pouvoir. L'État moderne est un Rechtsstaat ${ }^{33}$.

Cette intention de liberté objectivée dans la constitution - "le meilleur État est celui dans lequel règne le plus de liberté ${ }^{34}$ " - s'exprime avec plus de netteté encore dans le caractère franchement pluraliste de l'État moderne. En tant que tel, il n'a pas de base confessionnelle, ethnique ou linguistique. Il incorpore dans ses fondements philosophiques la notion chrétienne-kantienne de la personne comme fin en soi

29. J. D'HONDT, Hegel en son temps, Éditions sociales, 1968, p. 85.

30. Cf. J. Freund, L'essence du politique, Sirey, 1965, \#\# 81-83 ; Éric WeIL, Hegel et l'État, Vrin, 1950, $2^{\mathrm{c}}$ éd. 1966, p. 57 : "La où existe un document constitutionnel, il peut être plus ou moins bien rédigé, plus on moins clairement; il n'aura de force que s'il correspond à la constitution réelle, historique, à la constitution de la nation : d'abord, il faut prendre le mot de constiturion au sens qu'il a en physiologie."

31. Hegel, Principes... \#\# 215-216.

32. lbid., \# $274 \mathrm{R}$.

33. Il faudrait citer en entier la vigoureuse préface des Principes.

34. HeGEL, La raison dans l'bistoire, trad. K. Papaioannou, 10/18, p. 169 ; cf. E. WEIL, op. cit., p. 55 ss. 
et en tire les conséquences pratiques dans le cadre de l'État de droit : tolérance, liberté d'expression, mobilité sociale, égale accessibilité aux charges publiques, etc. "L'homme vaut parce qu'il est homme, non parce qu'il est juif, protestant, allemand ou italien ${ }^{35} \gg$. Bien que Hegel répercute certaines harmoniques platoniciennes, notamment celles reliant les classes sociales et certains modes d'être de la conscience, il s'écarte sur ce point fondamental du modèle monolithique de sa république $^{36}$. Il répudie de la même manière le modèle constantinien de la chrétienté médiévale d'une manière tout à fait personnelle et qui ne manque pas de profondeur. Selon lui, écrit E. Weil, « la sanctification du monde n'est pas la tâche d'un intérieur qui resterait éternellement intérieur, une tâche éternellement à accomplir et qui devrait rester éternellement non accomplie ; elle a été accomplie dans et par l'histoire, c'est une tâche qui appartient au passé, non à l'avenir. Le monde est devenu raisonnable quoiqu'il ne le soit pas entièrement ; ce qui reste du christianisme, c'est sa pensée (que la religion n'a saisie que dans le mode de la représentation), non la forme de la vie ${ }^{37}$ ».

Admirateur de Napoléon, navré de la nullité politique de l'Allemagne, il n'en critique pas moins l'excessive centralisation de la décision politique dans la France postrévolutionnaire. L'État doit rassembler les diverses parties de son territoire, mais dans le respect des particularismes régionaux et locaux.

Certes, Hegel ignore les développements ultérieurs de la «pleine modernité » (T. Parsons) : l'orientation vers le Welfare State, la dissociation de la propriété et du contrôle ou activité de gestion dans la grande entreprise ${ }^{38}$, le gonflement spectaculaire du tertiaire, l'impact énorme des mass media salué parfois comme quatrième pouvoir et agent de «la révolution culturelle », l'avènement de la société consommationniste, la récente révolution de l'enseignement «aussi

35. Hegel, Principes... \# 209.

36. Il perçoit le caractère déjà réactionnaire de ce type d'État dans le contexte de la Cité antique. Cf. S. Avineri, op. cit., p. 172, n. 53.

37. E. WEIL, "La sécularisation de l'action et de la pensée politiques à l'époque moderne " in Essais et conférences, t. II, Plon 1971, p. 30.

38. Selon R. GARAUDY, Marx avait déjà anticipé cette rupture, cf. L'alternative, R. Laffont, 1972 , p. 223. 
importante que le furent les révolutions industrielles et démocratiques " selon Parsons ${ }^{39}$, la contestation politique et culturelle $^{40}$, etc. Dans ses grandes lignes, cependant, c'est le réalisme de Hegel qui s'est avéré prophétique et ce sont les anticipations, prétendument progressistes des utopistes, qui se sont révélées dans l'ensemble régressives et archaïsantes.

Autant la représentation hégélienne de la modernité frappe par sa riche complexité, son sens dialectique du devenir concret progressant par la médiation de la négativité ellemême, autant les constructions abstraites de l'utopie brillent par leur passion quasi géométrique de l'ordre simple, uniforme, rigoureux, par leur horreur schizophrénique de l'historicité concrète et leur nostalgie de la petite communauté close et chaleureuse, repliée sur elle-même. Il est difficile d'imaginer un contraste plus complet. R. Ruyer nous prévenait déjà : "Cette philosophie est, sans conteste, la plus spécifiquement anti-utopique qui soit. Elle représente l'antipode de l'esprit utopique. Le moyen le plus radical d'empêcher l'homme de s'égarer dans les nuages des projets et des possibles idéaux, c'est évidemment de supprimer le monde même de l'idéal, comme distinct du réel ${ }^{41}$."

\section{Platon}

Cette orientation passéiste de la mentalité utopique se laisse déjà voir chez Platon. Dans la crise qui secoue l'ordre moral traditionnel, il réagit fortement au dissolvant de la sophistique. Dès lors que la foi jusque-là communément partagée est minée par la critique, elle ne peut plus être vécue comme pure foi, elle doit se fonder sur des bases nouvelles ou

39. op. cit., p. 100.

40. Encore ici la grille hégélienne paraît plus accordée à notre actualité que les prévisions marxistes. "Les étudiants, remarque B. de Jouvenel, ne sont pas des "travailleurs " au sens marxiste, comme ne participant pas au processus de production; ils ne sont pas des " exploités » au sens marxiste, comme n'étant pas employés par des capitalistes auxquels ils fourniraient des moyens de profit. Ils ne sont donc nullement dans une « condition objective " qui fasse entrer leur révolte comme nécessaire dans un schème déterminé par le conflit naturel des intérêts. Il me semble d'ailleurs qu'ils reviennent de Marx à Hegel, en situant le principe du changement non dans la lutte de classes mais dans un foyer d'idées. " «L'explosion estudiantine, "Analyse et Prévision, VI, (1968), p. 580.

41. R. RuYer, L'utopie et les utopies, P.U.F., 1950, p. 64 
disparaître comme sacré inconscient de lui-même par l'avènement d'un nouveau sacré : en définitive, seul un nouveau dieu peut chasser l'ancien. Cependant, dans les deux cas la restauration intégrale de l'ordre ancien est illusoire, mais il est possible de contenir pour un temps et dans certaines limites la dissolution du " conglomérat hérité ». Dans la débâcle culturelle de son époque, Platon s'attache à "stabiliser la situation au moyen d'une contre-réforme ". Sa riposte utopique présente un caractère conservateur, voire réactionnaire. Son image de la société idéale emprunte des éléments nettement situés dans le passé. "Bien que tous ses écrits soient probablement du IV siècle, observe E.R. Dodds, sa personnalité et ses perspectives furent formées au $\mathrm{V}^{\mathrm{e}}$, et ses premiers dialogues sont encore baignés de la lumière d'un monde et d'une société disparus. Le meilleur exemple à mon sens est le Protagoras, dont l'action se passe aux années merveilleuses d'avant la Grande Guerre ; dans son optimisme, sa mondanité cordiale, son utilitarisme candide et son Socrate qui n'est encore que " grandeur nature ", il semble être une reproduction essentiellement fidèle du pas$s^{42}$. " Du reste, sa nostalgie n'est pas seulement contemplative, elle cherche à modeler impérieusement la réalité : Platon est allé trois fois chez Denys II de Syracuse. Cette pensée qui semble révolutionnaire par sa volonté de "faire " la société à partir des exigences de l'Idée ne cherche en réalité qu'à l'arrêter dans la perfection d'un passé idéalisé. Par son anti-historicisme foncier, elle manque la dialectique concrète de la tradition et du progrès : la tradition s'en trouve comme dévitalisée et figée dans une synchronie définitive, répétitive, et « le mouvement qui déplace les lignes" ne saurait s'exercer à l'intérieur d'une société dont chaque élément ne peut sortir de son lieu sans attenter à l'architecture de l'ensemble. Une fois les pièces du puzzle social bien assemblées, l'ordre ne peut être que maintenu, reproduit, célébré. D’autre part, l'intégrité de l'ordre et des mours de la cité est préservée par la séparation d'avec le monde extérieur. Dans les Lois, Platon ne permet aucun voyage à l'étranger sinon pour le service public. Les citoyens-voyageurs sont sélectionnés ; une fois rentrés, ils sont tenus d'exposer à la jeunesse combien ils ont trouvé inférieures

42. E. R. DoDDs, Les Grecs et l'irrationnel, Aubier, 1965, p. 200-1. 
les institutions des autres pays. L'autarcie économique et l'autarcie culturelle se renforcent mutuellement. Par le biais du commerce, la cohésion morale du groupe risque toujours de se relâcher. C'est pourquoi il existe deux monnaies, l'une pour l'usage intérieur, l'autre pour les contacts avec l'étranger ${ }^{43}$. Sans entrer plus avant dans les détails, on peut ranger les principales cuvres sociales et politiques de Platon en deux catégories également impliquées avec la problématique de l'utopie, soit par le contenu, soit par l'intention. "Si le contenu de la République du Politique et des Lois est déjà spécifiquement utopique, le vrai roman utopique, le chant des sirènes qui a ensorcelé pour des siècles les imaginations, c'est dans le Timée et le Critias qu'il se trouve, avec l'histoire de l'Atlantide ${ }^{44}$. »

\section{More}

De Platon à More la continuité est évidente. Comme chez Platon, le projet d'une communauté juste et harmonieuse est chargé de nostalgie. Comme dans beaucoup d'utopies, la rupture avec le temps de l'aliénation et du désordre est marquée non seulement par une nouvelle constitution juridique mais aussi par une constitution géographique. L'immunité vis-à-vis des retours pernicieux de l'influence extérieure est garantie par la coupure symbolique avec le continent. L'utopie est une île de forme et de dimensions déterminées. Au centre, Amaurote, la capitale, dirige ses 54 villes régulièrement réparties à travers le pays. Des rues de vingt pieds sont de part et d'autre bordées d'édifices; les quartiers résidentiels quadrillent le territoire avec leurs maisons uniformes assorties à l'avant de parterres et de fleurs, à l'arrière de petits jardins. Chaque quartier dispose d'un marché, d'une place, d'un temple. Chaque rue comporte un hôtel servant à la fois de demeure aux magistrats et de restaurant collertif. $\grave{A}$ chaque ville est attribué un territoire d'environ vingt kilomètres de côté. Chaque famille se compose de quarante membres. La

\footnotetext{
43. Ce trait est tellement typique de l'utopie que, selon H. Freyer, L'État commercial fermé de Fichte peut être considéré comme le "sommet de l'histoire de l'Utopie ". Cité in W.E. MÜhlmanN, Messianismes révolutionnaires du tiers monde, NRF, 1968, p. 304.

44. R. RUYFR, op. cit., p. 137.
} 
population ne dépasse pas six mille familles. Si la chose s'avère nécessaire, on fonde une colonie. Nul ne peut quitter sa ville sans une autorisation formelle du conseil municipal. Des contrôles rigoureux précèdent et suivent le déplacement. Mais « celui qui, de son propre mouvement, se permet de franchir les limites de sa province, est traité en criminel; pris sans le congé du prince, il est ramené comme un déserteur et sévèrement puni. En cas de récidive, il perd la libertét ${ }^{45}$.

Chaque journée est réglée par un horaire rigoureux. Chacun dans son alvéole travaille, étudie, se détend, prie selon des prescriptions spécifiques. Sanctions et récompenses renforcent l'ordre général. Les lois simples et peu nombreuses se présentent comme des conséquences directes des principes moraux universels.

Le pouvoir temporel, fortement structuré, incorpore une certaine discussion publique. À côté de ce pouvoir, Utopie s'est donné un pouvoir spirituel qui ne peut contraindre personne, mais qui peut réprimander et excommunier les « hommes d'une perversité scandaleuse ». Cependant, ce rejet "les note d'infamie, torture leur conscience de mille craintes religieuses, et même elle ne les laisse pas tranquilles sur la sûreté de leurs personnes puisque s'ils ne se hâtent pas de donner aux prêtres des marques de vrai repentir, le sénat les fait arrêter et leur applique la peine des impies ${ }^{46} \gg$. Toutes les confessions religieuses sont tolérées. Néanmoins, cette tolérance ne s'applique pas aux "matérialistes" négateurs de l'immortalité de l'âme et de la Providence. En effet, les Utopiens « ne donnent pas le nom d'homme à celui qui nie les vérités, et qui ravale la nature sublime de son âme à la vile condition d'un corps de bête ; à plus forte raison ne l'honorentils pas du titre de citoyen, ajoute More, persuadés que, s'il n'était pas enchaîné par la crainte, il foulerait aux pieds, comme un flocon de neige, les mœurs et les institutions sociales. (. . .) À ces matérialistes, on ne rend aucun honneur, on ne communique aucune magistrature, aucune fonction publique. On les méprise comme des êtres d'une nature inerte

45. T. MORE, L'Utopie, Éditions sociales, 1966, p. 137.

46. Ibid., p. 189-90. 
et impuissante ${ }^{47} »$. De plus, c'est au clergé que revient la charge de l'éducation. Comme on peut le deviner, ses premiers soins vont «à l'enseignement de la morale et de la vertu, plutôt qu’à celui de la science et des lettres ${ }^{48} »$. Ce n'est pas assez que les prêtres se soucient des bonnes mœurs de leurs ouailles, les magistrats étendent leur sollicitude aux rapports intimes de l'homme et de la femme. Comme chez Platon et Campanella, les fiancés doivent se contempler nus de la tête aux pieds sous le regard d'un gardien ou d'une matrone. L'adultère est puni du plus dur esclavage. La récidive est punie de mort. Ces magistrats que l'on appelle d'ailleurs pères réservent aux déviants "indigènes » des châtiments d'une rigueur particulière. Selon toute apparence, ils ont droit à un esclavage d'exception, car la peine ordinaire, même des plus grands crimes, est l'esclavage. "Les Utopiens croient que l'esclavage n'est pas moins terrible pour les scélérats que la mort, et qu'en outre il est plus avantageux à l'État (. . .) Quand les condamnés esclaves se révoltent, on les tue comme des bêtes féroces et indomptables que la chaîne et la prison ne peuvent contenir ${ }^{49}$." Si l'on peut trouver des fanatismes plus sombres et des moralismes plus tristes dans le cortège des utopies, il faut avouer que la tolérance et l'épicurisme de More sont au demeurant fort modérés ${ }^{50}$.

L'économie planifiée, organisée par l'État, ne laisse personne errer à l'aventure ou parasiter le corps social. De la sorte une journée de travail de six heures suffit à satisfaire les besoins frugaux de tous. L'activité principale demeure l'agriculture, bien que chaque citoyen puisse apprendre parallèlement un métier : tissage, céramique, etc. Comme il n'y a ni propriété privée, ni monnaie, ni privilège de l'or (ce vil métal sert à la fabrication de vases de nuit), ni commerce intérieur, chacun s'approvisionne aux magasins généraux selon ses be-

47. $\quad$ lbid., p. 185

48. Ibid., p. 190

49. lbid., p. $166,160,165$.

50. M. de GANDILLAC observe avec pertinence : "Exactement contemporaine du Prince, l'Utopie, en cerraines de ses pages, n'est guère moins cynique, mais justifie par des fins haurement morales l'emploi conjugué de la propagande er de la violence. Lorsque les paysans allemands, de manière plus ingénue, vont passer à l'action, More oubliera vite son manifeste révolutionnaire. Sur le papier du moins, il est pourtant l'ancêtre de tous les organisateurs d' " armées rouges ", de tous les spécialistes d'agit-prop. "In Histoire de la philosophie, t. II, Pléiade, NRF, p. 201. 
soins. En termes hégéliens, la société civile et la famille ne font qu'un sous la tutelle paternelle de l'État. "Ainsi la république utopienne tout entière est comme une seule et même famille ${ }^{51}$. " Chaque élément du tout social, à sa place, joue le rôle qu'on attend de lui.

Préservant jalousement leur splendide isolement par la corruption, par la trahison, par la terreur semées chez l'ennemi, les Utopiens jouissent chez eux d'un bonheur paisible et monotone. Telle une horloge douée d'un mouvement perpétuel, le pays d'Utopie répète dans un rythme circulaire la perfection de son ordre. Le déménagement universel tous les dix ans, l'assignation de nouvelles maisons par voie de tirage au sort, n'est-ce pas un assez beau symbole d'un changement sans conséquences et de l'interchangeabilité des personnes autant que des objets ? La dialectique du progrès est ruinée dans son principe : société parfaite, l'Utopie ne peut se dépasser qu'en s'étendant par la contagion de l'exemple à l'ensemble de l'humanité.

Thomas More a réussi ce tour de force d'être successivement annexé à l'héritage spirituel de l'humanisme, du catholicisme et du socialisme ${ }^{52}$. Mérite-t-il ces honneurs multiples? Est-il du côté de l'Ordre ou du côté du Progrès, si tant est que l'on puisse discriminer fermement les tenants de l'un et de l'autre camp? Avec le recul du temps, il semble cependant que l'investigation historique dissipe l'ambiguïté. M. Horkheimer, peu suspect de complaisance à l'égard du capitalisme, écrit pourtant : "La propriété collective, les rapports d'existence basés sur l'égalité tels que les utopistes les exigeaient, auraient signifié la mort de la civilisation. C'est pourquoi en comparaison de More et de Campanella, Machiavel et Hobbes apparaissent comme progressistes ${ }^{53}$. » De son côté, R. Ruyer observe: "Morus réchauffe un vieil idéal de communauté morale et de stagnation économique à la veille des guerres

51. Ibid., p. 138.

52. A. CIORANESCU, L'avenir du passé, utopie et littérature, NRF, 1972, p. 271 : "More était pour Lénine ce qu'Homère avair éré pour Alexandre et Plutarque pour Napoléon. " Quant à Kautsky, il n'hésitait pas à voir en lui "le premier socialiste moderne " (p. 251).

53. Max Horkheimer, Les débuts de la philosophie bourgeoise de l'bistoire, Payot, 1974, p. 103 
religieuses et de l'expansion économique moderne ${ }^{54}$." A. Cioranescu ne juge pas autrement: "Plutôt qu'ami du progrès, More fait figure de réactionnaire. Il s'oppose au capitalisme, qui avait des inconvénients déjà visibles, mais qui était alors vraiment le progrès. À la place d'une société corrompue et matérialiste il propose un idéal de justice, qui n'est qu'un retour aux sources. On vante la clairvoyance avec laquelle il défend les petits exploitants agricoles contre les méfaits des éleveurs, principaux fournisseurs de l'industrie ; et sa phrase sur les moutons qui dévorent les hommes est dans tous les manuels. C'est là une affaire qu'il devait connaître parfaitement, puisque c'était précisément l'objet de sa mission en Flandre ; mais on n'a pas observé que là encore il défend des positions conservatrices puisque, dans le grand procès historique entre l'agriculture et l'industrie, il prend position pour la première. " (. . ) «Il ne s'agit pas d'abolir le passé, mais de dégager la tradition de la routine et de l'abus. Il n'est révolutionnaire que dans la mesure où l'on veut admettre que l'on peut faire du neuf avec du vieux exclusivement. " Et encore : " La nostalgie communiste, dans la vision de More et de toutes les utopies en général, est bien une nostalgie, c'est-à-dire un retour en arrière et une solution a posteriori; comme toutes les nostalgies, elle est en même temps l'application incorrecte d'une solution dépassée ${ }^{55}$. »

\section{Campanella}

La démonstration serait encore plus aisée avec $L a$ cité $d u$ soleil de Campanella, que l'on a pu appeler « un rétrograde forcené ${ }^{56} »$. Autant l'espace est suraménagé selon les requêtes géométriques de l'esprit et l'horaire quotidien minutieusement déterminé, autant la collectivité solarienne se barricade contre les aléas d'un devenir non calculé en vue de la connaissance intégrale, de la maîtrise du torrent tumultueux de la vie, et de l'Amour universel. À l'inverse de la pluralisation et de la différenciation des ordres de vérité et d'existence, le Domini-

54. R. RUYER, op. cit., p. 53.

55. A. Cioranescu, op. cit., p. $105,55$.

56. Ibid., p. 255. 
cain calabrais procède à une involution régressive vers la confusion primitive du spirituel et du temporel : « Ils ont un Prêtre souverain qu'ils appellent Soleil et que nous pouvons nommer le Métaphysicien : il commande à tous aussi bien dans le spirituel que dans le temporel, et pour toutes les affaires il détient un pouvoir discrétionnaire. Il est assisté par trois princes : Pon, Sin, Mor, c'est-à-dire : Pouvoir, Sagesse et Amour ${ }^{57}$. Tout converge vers une sorte de grande famille organisée selon le modèle des communautés religieuses de l'époque. Campanella l'a bien vu, l'individu, le couple, la famille constituent naturellement des noyaux de résistance à l'homogénéisation totale : "On dit chez eux que la propriété provient du fait que l'on vit dans des maisons séparées avec sa femme et ses enfants et que cela fait naître l'égoïsme. Et pour faire exceller son fils par la richesse ou par la dignité, ou lui laisser un héritage, un chacun devient ouvertement rapace s'il ne craint rien grâce à sa puissance, ou un avare, un rusé, un hypocrite, s'il n'a aucun pouvoir. Mais supprimez l'amourpropre, il ne subsiste que l'amour universel ${ }^{58}$. " C'est à cela précisément que s'ordonne toute l'entreprise. Le moine italien ne recule devant aucune institution, même délirante, qui soit propre à déraciner l'amour-propre.

Sous la direction de Mor, les magistrats contrôlent les accouplements et les générations. Les maîtres font la sélection des garçons et des filles au moment de séances de lutte qu'ils pratiquent nus. (En règle générale, ils ne peuvent faire l'amour avant dix-neuf ans pour les filles, avant vingt et un ans pour les garçons). Le soir prescrit, après un bon bain, prière et digestion faites, ils se mettent au lit dans des cellules séparées jusqu’à l'heure où ils doivent s'aimer. Au moment précis, déterminé par l'astrologue et le médecin, la matrone ouvre la porte qui relie les deux chambres. Dès deux ans, les enfants

57. T. Campanella, la Cité du Soleil, trad. A. Tripet, Droz, 1972, p. 6. Sur l'interpénétration de la religion et du pouvoir dans les sociétés archaiques on lira avec profit G. BALANDIER, Antbropologie politique, PUF, 1969, chap. V. Il y rapporte la formule paradoxale de Luc de Heusch: "La science politique relève de l'histoire comparée des religions. "Rien n'est moins sûr du reste que ce lien se défasse tout à fait dans les régimes modernes. R. RuYer écrit : "Il n'y a pas d'État vraiment lä̈que, et quand l'État se sépare de l'Église, c'est toujours pour épouser au plus vite une autre Église. " Les nuisances idéologiques, Calmann-Lévy, 1972, p. 179.

58. Ibid., p. 11 . 
sont pris en charge par l'État, éduqués, instruits. À dix ans, on les voit déjà au travail.

Comme chez More, les déménagements visent à arracher le sentiment de propriété mais les temps ont changé : désormais, c'est tous les six mois que les Solariens échangent leurs logis.

Homme de la Renaissance, Campanella veut des hommes instruits. Les murs sont recouverts de figures géométriques, d'images d'animaux et de plantes, de reproductions des mécaniques créées par l'ingéniosité humaine.

More s'efforçait encore de décourager les ambitieux qui jettent l'instabilité dans la république : "Celui qui brigue une seule magistrature perd tout espoir d'en exercer jamais aucune ${ }^{59}$." Cette précaution devient inutile: les magistrats sont assez clairvoyants pour reconnaître la supériorité et assez sages pour lui céder la place.

Mais ici encore l'ironie de l'Histoire trouve une matière de choix pour exciter sa verve : les conséquences ont été à l'opposé des intentions de l'utopiste. «Campanella, qui . voulait la grandeur de l'Église, fut l'un de ceux qui contribuèrent à en saper les fondements politiques ${ }^{60}$. »

\section{Rousseau}

La carrière historique de Rousseau se prête également à ce genre de remarques. Certes, si l'on veut prendre sa pensée sociale et politique dans la totalité de ses expressions, il faudrait une étude longue et fort nuancée pour clarifier l'articulation exacte de ses implications utopiques et de ses propositions réalistes ${ }^{61}$. Néanmoins, nous pouvons relever quelques points utiles pour notre propos.

Avant Hegel, Rousseau a vivement ressenti la dislocation de la petite communauté nationale sous le choc du mercanti-

59. T. MORE, op. cit., p. 166.

60. A. Cioranescu, op. cit., p. 143.

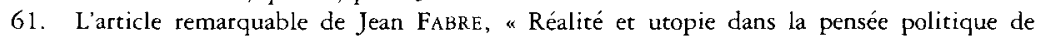
Rousseau ", Annales Jean-Jacques Rousseau, t. XXXV, 1959-62, p. 181-216, demeure fondamental. 
lisme et de l'industrialisme modernes portés par la montée de la bourgeoisie. À ses yeux, il était urgent de repolitiser la problématique de la liberté émiettée dans des directions diverses : théologie, épistémologie, psychologie. La socialisation anarchique de l'économie appelait la recréation d'un éthos communautaire que seul l'État pouvait entreprendre en gardant ses distances vis-à-vis du maelström de la société bourgeoise. "Les anciens Politiques parlaient sans cesse de mœurs et de vertus ; les nôtres ne parlent que de commerce et d'argent", déplore Rousseau ${ }^{62}$. Mais au lieu de chercher un "dépassement " qui préserve les acquis positifs de la modernité au-delà de ses limitations bourgeoises, à la manière de Hegel, il institua un violent réquisitoire contre la société moderne au nom d'une double utopie implicite : celle de l'Âge d'or qui coïncide avec la première notion de l'état de nature et celle de la vertueuse Sparte selon l'image qu'en donne Plutarque. Dans les deux cas cependant le fondement de l'imagination critique emprunte ses éléments à un Passé rêvé ou mythologisé : nostalgie d'un état de nature prépolitique, nostalgie de la cité antique. La représentation préhistorique de l'état originel et la position a priori de l'originaire mêlent ici leurs eaux. Dans cet état de sauvagerie, l'homme ne se distingue pas de l'animal.

Selon « l'utopie " de l'Âge d'or, Rousseau nous peint un état tout à fait abstrait, un présent perpétuel. Aucun intervalle ne sépare les besoins et les désirs naturels des ressources de la nature. Ignorance, innocence, oisiveté, autosuffisance, immédiateté, tel est l'état de nature selon la vérité du cour. Mais l'homme est seul dans cette nature généreuse : "Je le vois se rassasiant sous un chesne, se désaltérant au premier Ruisseau, trouvant son lit au pied du même arbre qui lui a fourni son repas, et voilà ses besoins satisfaits ${ }^{63}$. "Par cette évocation poétique, l'œuvre se sépare de l'utopie proprement dite qui construit d'une manière détaillée un monde autre dans lequel les hommes pourraient vivre bien qu'ils refusent toujours de le faire. En effet, le centre brûlant de l'utopie se nourrit de l'idée

62. J.-J. Rousseau, Discours sur les arts et les sciences, In Oeutres completes, t. III, Pléiade, NRF, 1966, p. 19.

63. J.-J. Rousseau, Discours sur l'origine de l'inégalité, O.C. t. III, p. 135 
d'une communauté authentique, fraternelle ${ }^{64}$. D'autre part, le vague de cette dérive mythico-poétique nous dépayse selon un axe para-utopique. "Peut-être, écrit R. Barthes, l'imagination $d u$ détail est-elle ce qui définit spécifiquement l'Utopie (par opposition à la science politique); ce serait logique, puisque le détail est fantasmatique et accomplit à ce titre, le plaisir même du Désir ${ }^{65}$. » Il reste cependant que l'hypothèse de la plénitude des origines fournit un fondement idéal à la condamnation du cours actuel de l'histoire et participe de l'irréalisation utopique.

Sparte nous ramène en un lieu et en un temps déterminés. Pourtant, ce n'est pas à la cité telle qu'elle fut que Rousseau s'intéresse mais à la cité sans murailles, au mythe de la communauté unie dans la vertu républicaine. Corrélativement, son mépris pour Athènes, la ville ouverte aux échanges extérieurs, la capitale de la culture savante et du bon goût est une charge à peine voilée contre la société des beaux esprits de Paris, vaniteuse, hypocrite, corrompue et divisée par les rivalités. Nul doute qu'il ne pense également à Londres, la capitale de l'argent et du commerce, "société de tête » de l'Europe moderne. L'image des vertus hérö̈ques de Sparte lui sert d'antithèse pour mesurer l'ampleur de la déchéance historique. Dans le miroir de cette utopie pratiquée, les hommes de son temps peuvent contempler la perte du véritable esprit civique et la dégradation de leur existence divisée. Des petites confréries fermées, en effet, elle a la rigueur, la simplicité, l'immobilité, le sens de la fraternité médiatisé par un patriotisme farouchement conservateur. À la complexité mouvante et sans âme des grandes villes, il oppose l'étroite cohésion morale et la discipline militaire; à la multiplication hédoniste des nouveaux besoins rendus possibles

64. Parfois cette hantise s'exhibe de la manière la plus explicite. À partir de la troisième édition de son Voyage en Icarie, Cabet dispose les deux mots fraternité et bonbeur commun sur la page frontispice ; des capitales en caractères gras sont utilisées.

65. R. BARTHES, Sade, Fourier, Loyola, Seuil, 1971, p. 110.

Dans la même veine, F. LAPLANTINE considère " que la conception même de l'utopie est typiquement occidentale et plus précisément hellénique et quà sa connaissance aucun groupe ethnique ayant évolué en dehors de notre sphère d'influence n'a projeté son imagination dans la direction de la rationalité utopique et mathématique, mais toujours dans celle de l'irrationalité mythique $»$. Les trois voix de l'imaginaire, Éditions universitaires, 1974, p. 191. 
sur une nouvelle échelle par le progrès des connaissances et des techniques, il oppose l'austérité et la frugalité antiques ; au cosmopolitisme suprapolitique des Lumières, il oppose une « ivresse patriotique » fort ambiguë ${ }^{66}$; à la division du travail et au ressentiment social, il oppose le paisible village de Neufchâtel où chacun sur son lopin de terre, « renfermé bien chaudement, avec sa nombreuse famille, dans sa jolie et propre maison de bois, qu'il a bâtie lui-même, s'occupe de mille travaux amusants, qui chassent l'ennui de son asile, et ajoutent à son bien-être. Jamais menuisier, serrurier, vitrier, tourneur de profession, précise-t-il, n'entre dans le pays ; tous le sont pour eux-mêmes, aucun ne l'est pour autrui ${ }^{67} \%$. À l'intérieur même de son cuvre, on peut s'interroger sur le contraste entre l'idéal démocratique du Contrat social et la structure encore féodale de la communauté de Clarens, ainsi que sur la double idéalisation de la vertu antique et de l'attitude plus moderne de M. de Wolmar, bienveillant à ses voisins, cultivant son verger, loin de l'agitation et des clameurs du forum ${ }^{68}$.

Ce n'est pas que Rousseau veuille ressusciter Sparte ou « ramener les peuples nombreux ni les grands États à leur première simplicité, mais seulement arrêter s'il était possible le progrès de ceux dont la petitesse et la situation les ont préservés d'une marche aussi rapide vers la perfection de la société et vers la détérioration de l'espèce ${ }^{69} \gg$. On le voit, son intense moralisme politique ne l'empêche pas de jauger avec lucidité les conditions objectives de sa réalisation.

Bien que le Contrat social se présente à première vue comme une cuvre doctrinale fort abstraite (elle porte le sous-titre de Principes du droit politique), elle n'en comporte pas moins des parties théoriques sur lesquelles l'analyse politique

66. Cf. R. Derathé, "Patriotisme et nationalisme au XVIII siècle », in L'idée de nation, PUF, 1969.

67. J.-J. Rousseau, Lettre à M. D'Alembert sur son_article Genève, Garnier-Flammarion, 1967, p. 134. Sur ces pages si franchement antismithéennes, lire l'excellent article de $B$. de Jouvenel, "Rousseau, évolutionniste, pessimiste ", APP, $n^{\circ} 5,1965$.

68. Cf. B. de JOUvEnEL, "Théorie des formes de gouvernement chez. Rousseau ", Le Contrat social, nov.-déc., 1962.

Sur l'intention foncièrement conservatrice de Rousseau et son action révolutionnaire, on lira avec profit l'article de Iring FETSCHER, APP, n5, 1965.

69. j.-J. Rousseau, Rousseau juge de Jean-Jacques, je dialogue, O.C., t.I, p. 935. 
contemporaine peut s'appliquer avec profit ${ }^{70}$. Inversement, les textes trop méconnus sur la Pologne et la Corse contiennent des stipulations concrètes qui rappellent l'aspect extérieur de l'utopie comme genre littéraire ${ }^{71}$. On pourrait parler d'utopisme sans utopie. En tout cas, on retrouve chez lui l'aspiration utopique de la communauté petite, fermée, autarcique, unanime jusqu'à l'intolérance et la xénophobie en même temps qu'un réalisme psychopolitique aigu. À la suite d'Aristote et de Montesquieu, Rousseau le reconnaît avec une netteté parfaite : le changement de dimension de l'ensemble humain est identiquement un changement de nature. Il est vain d'espérer obtenir l'éthos communautaire propice au développement de l'esprit public dans les vastes agglomérations urbaines. C'est pourquoi seule la Corse lui paraît finalement susceptible d'actualiser approximativement son idéal politique. De là le verdict rigoureux : "L'institution publique n'existe plus et ne peut plus exister; parce qu'où il n'y a plus de patrie il ne peut plus y avoir de citoyens ${ }^{72}$."

En reprenant à son compte l'idée de paix perpétuelle comme souverain bien politique, Kant avait fait figure de transfuge du camp des philosophes pour rejoindre l'abbé de Saint-Pierre qui, selon Rousseau, avait atteint la célébrité par le ridicule. Paradoxalement, c'était le rêveur solitaire qui se montrait fort sceptique sur la base de considérations empiriques réalistes. Cependant, Kant demeurait fidèle à la notion classique de la paix comme but spécifique du politique. Sa tâche n'est pas de régénérer l'humanité, mais de la conserver. Il n'est pas nécessaire que les hommes soient vertueux pour vivre en paix, il leur suffit d'être intelligents. Il est possible de faire vivre ensemble une bande de démons pourvu qu'ils sachent calculer leur intérêt à long terme. Bref, le problème politique est soluble indépendamment de la morale et de la religion.

70. Cf. B. de JOUVEneL, ant. cit.

71. Judith N. SHKLAR, Men and Citizens, Cambridge, 1969, p. 14-5: "Indeed, the pseudo-realism of his Polish plan, with its endless descriptions of the details of organization and ritual, make it the most visionary, pejoratively utopian, of his works. "

72. J.J. Roussfau, Emile, O.C., t. IV, 1969 , p. 250. 
Mais la conception rousseauiste de la Volonté Générale et les institutions qui y sont rattachées - suffrages, censure, religion civile, "démocratie directe ", législateur-guide, etc. - impliquent la volonté de créer un homme nouveau dépouillé des inclinations de l'amour-propre et totalement voué à « l'intérêt commun ». C'est pourquoi on a pu dire " qu'il y a du Loyola dans Rousseau ${ }^{73}$ ». Le citoyen doit pouvoir en arriver, par vertu, à aimer la Volonté Générale avec la même ardeur que l'individu s'aime naturellement lui-même. "Les bonnes institutions sociales, écrit-il, sont celles qui savent le mieux dénaturer l'homme, lui ôter son existence absolue pour lui en donner une relative, et transporter le moi dans l'unité commune; en sorte que chaque particulier ne se croye plus un, mais partie de l'unité, et ne soit plus sensible que dans le tout. Un citoyen de Rome n'était ni Cä̈us ni Lucius : c'était un Romain : même il aimait la patrie exclusivement à lui ${ }^{74}$." Dès lors, il ne s'agit plus seulement de régler l'extériorité des actions mais également les opinions et les sentiments intimes des individus. Rousseau rêve d'une République qui nourrirait les cœurs autant qu'elle protégerait ses sujets.

Avant lui, Hobbes avait "osé proposer de réunir les deux têtes de l'aigle, et de tout ramener à l'unité politique ${ }^{75}$ ", mais l'individu de Hobbes, calculateur et égoïste, gardait encore le vif sentiment d'être un tout pour lui-même et liait son obéissance à la protection efficace du Souverain : il ne lui demandait point de s'immoler sur l'autel de la Volonté Générale. Rousseau ainsi que les Jacobins d'hier et d'aujourd'hui retrouvent Platon et sa "laconomanie », selon l'expression d'Aristophane: "Tout ce qui rompt l'unité sociale ne vaut rien ${ }^{76}$. " L'existence s'en trouve intégralement politisée. La culture de l'esprit et des sentiments ne saurait obéir à une dialectique indépendante de l'intégration politique. La religion

73. G. BuRdeAu, "Le citoyen selon Rousseau ", in Études sur le contrat social, Les belles lettres, Dijon, 1964, p. 220.

74. J.-J. Roussfau, Émile, p. 249. Le verbe extrêmement énergique " dénaturer " n'est pas employé légèrement; il revient à la page suivante sous sa plume avec une insistance voulue : "Platon n'a fait qu'épurer le coeur ; Lycurgue l'a dénaturé. " Cf. aussi CS, II, 7.

75. J-J. Rousseau, Du contrat social, IV, 8.

76. Ibid. 
nationale sacralise la cohésion de la communauté élevée à la dignité de Souverain Bien. Les pouvoirs publics ne se donnent plus pour tâche propre de préserver un minimum de sécurité et de paix grâce auquel l'individu peut vivre et agir selon des idéaux et des valeurs non immédiatement politiques : science, art, morale, religion, économie. Dorénavant, ils prétendent apporter salut et bonheur avec les moyens dont ils disposent la force et la ruse. Il n'est plus seulement demandé au citoyen de participer à l'édification d'une communauté plus juste et plus fraternelle dans le respect des lois et des rites superficiels de la simple civilité ; il faut encore la reddition intérieure de chacun à la Volonté Générale. Toute dissidence, tout repliement égoïste est une sécession intolérable. L'opinant minoritaire doit reconnâttre son erreur et rallier le sentiment général ; l'indifférent ou le profiteur doit s'attendre «qu'on le forcera à être libre ${ }^{77} »$. Cette surcharge eschatologique de la téléologie spécifique du politique, typique de l'utopisme, l'histoire nous l'a abondamment montré, était grosse de la Terreur révolutionnaire.

Comme tous les utopistes, Rousseau ne s'embarrasse guère des moyens efficaces de la réalisation de son intention. Cette référence négative aux moyens est tellement essentielle que Mühlmann l'inclut dans sa définition de l'utopie : «Par utopie nous entendons tout projet de communauté humaine conçu sans prévision méthodique des moyens de la réaliser ${ }^{78}$. " Mais en même temps, il est le premier à convenir de l'immédiateté abstraite de sa pensée et, à la limite, de son caractère circulaire, suicidaire. "Pour qu'un peuple naissant pût gouter les saines maximes de la politique et suivre les règles fondamentales de la raison d'État, il faudroit que l'effet pût devenir cause, que l'esprit social, qui doit être l'ouvrage de l'institution, présidât à l'institution même ; et que les hommes fussent avant les loix ce qu'ils doivent devenir par elles ${ }^{79}$. "Le passage subit et volontaire de la sauvagerie naturelle à l'ordre politique le plus sévère qui soit ne peut être parcouru que par

77. Ibid., I, 7

78. W.E. MÜHlMaNN, op. cit., p. 301.

79. J.-J. ROUSseau, Du contrat social, II, 7. 
l'imagination d'un « miracle ${ }^{80}$. Et comme les « réalistes », Rousseau ne compte pas sur les ressources morales de ses contemporains. S'il répudie la voie révolutionnaire, il demeure loin de la stupéfiante nä̈veté stratégique d'un Fourier par exemple $^{81}$. Quant au parlementarisme, on sait qu'il heurte son exigeante conception de la liberté : "Le peuple anglois pense être libre, il se trompe fort ; il ne l'est que durant l'élection des membres du Parlement: sitôt qu'ils sont élus, il est esclave, il n'est rien ${ }^{82}$. »

Ce refus des médiations institutionnelles de la liberté concrète, cette hostilité à l'égard du régime « constitutionnelpluraliste » séparent radicalement la liberté des Anciens et celle des Modernes. Autant Rousseau rejette tout corps intermédiaire entre le citoyen et le Souverain ${ }^{83}$, autant Hegel s'appuie sur les corporations, d'une part, pour éviter la tyrannie du Prince et les émotions de la foule, de l'autre, pour objectiver la nécessaire différenciation de la synthèse moderne des volontés. Comme Rousseau, Hegel critique l'individualisme du schéma contractualiste, mais il ne régresse pas non plus vers le modèle spartiate d'une intégration sans différences. À cet égard, sa compréhension inexacte de la Volonté Générale (dans le \# 258 des Principes) n'invalide pas le "dépassement" original qu'il effectue de ces vérités partielles. Hegel a vraiment tracé la voie à une philosophie politique qui récapitule en elle, critiquement et dialectiquement, la totalité de l'histoire. Son réalisme ouvert à l'avenir court-circuite également l'apriorisme abstrait des utopies et l'obturation " intempestive " du conservatisme, fermé au cheminement progressif et dialectique de l'Esprit.

80. Voir les notes manuscrites de Rousseau rapportées par $\mathbf{B}$. de Jouvenel dans son édition du Contrat.

81. Cf. C. LeHOUCK, Fourier aujourd'bui, Denoël, 1966, p. 113-129.

82. J.-J. ROUSSEAU, Du contrat social, III, 15.

83. Là encore, Rousseau ne se fait pas faute de transgresser ses propres principes quand la situątion l'exige. C'est ainsi qu'il admet la nécessité de représentants dans le cas de la Pologne. Cf. là-dessus la fine analyse de J. -C. PARIENTE, « Le rationalisme appliqué de Rousseau ", in Hommage à Jean Hyppolite, 1971. 
Nous partons généralement avec un préjugé favorable à l'égard de l'utopie. Le langage de l'objectivité ne réclame-t-il pas un effort auquel nul ne peut s'astreindre continûment ? La lumière crue de l'intellect chasse les dieux et les démons, mais dissipe aussi le clair-obscur des rêveries où le cour se régénère. Bachelard nous l'a appris, si la métaphore est la voie royale de la poésie, elle est le péril de l'esprit scientifique. L'imagination utopique restaure les droits de la fantaisie en ce siècle de fer. Pour vivre et pour agir, le langage de l'espérance n'est-il pas aussi nécessaire que les minutieuses procédures de la méthode scientifique? En tout cas, le doute, si vital pour le progrès théorique, est source d'hésitation et de paralysie pour l'action. Et puis, les utopistes n'ont-ils pas forgé des rêves qui ont finalement trouvé le chemin de la réalité ?

Pourtant, cette présomption résiste mal à la confrontation de l'histoire. À supposer que la plupart de ces phantasmes soient réalisables dans un avenir indéterminé, il est permis de douter que beaucoup parmi nous soient tentés de s'embarquer sans retour vers ces pays de rêve. Ce que l'histoire a réalisé des anticipations utopiques appartient plutôt à la science-fiction. Ici, l'imagination s'enchante des prouesses de la science et de la technique et rebondit sur ses virtualités pratiquement illimitées. Effectivement, c'est à ce niveau que la réalité du progrès s'impose d'une manière irrécusable. Mais la pointe de l'utopie dépasse les effets engendrés par la virtuosité technique; elle n'est pas non plus dans l'invention institutionnelle comme telle ; elle réside dans le projet d'une nouvelle communauté humaine, affranchie de la pression tyrannique du besoin et des passions mesquines de l'individu; elle planifie un ordre nouveau où l'accomplissement intégral de chacun s'associe étroitement à l'intense fraternité de l'ensemble. À la limite, la visée utopique se confond avec la visée religieuse d'une humanité régénérée, totalement réconciliée, achevée. En d'autres termes, c'est au niveau eschatologique des fins ultimes que l'utopiste opère. À l'inverse du praticien réaliste de la politique, quelque peu démagogue, essentiellement opportuniste, composant habilement avec les hommes tels qu'ils sont, l'utopiste construit un autre monde, un antimonde à partir de l'idée de la cité telle qu'elle devrait être selon lui. Révolté par 
la société existante, il se précipite d'emblée, en imagination, vers une autre société qui serait, elle, l'expression transparente des vraies valeurs. Mais en même temps, il ne se perd pas dans la contemplation d'une humanité fictive ou dans le vague souhait d'une nouvelle société, il veut effectuer son espérance. Platon est allé trois fois à Syracuse. More n'a pas lui-même tenté de réaliser son utopie comme le feront plus tard Cabet et Owen (encore qu'on ne connaisse pas très précisément son rôle dans la tentative avortée de son beau-frère, John Rastell). Il reste que son engagement au service du roi n'avait rien d'inconditionnel. Pendant près de trois ans, More essaya de concilier conscience et politique, mais, en 1532, il vit clairement que l'homme de l'utopie ne pouvait avoir raison de l'homme de l'Histoire et démissionna. On connaît la suite : il eut la tête tranchée en 1535. Campanella a passé vingt-sept ans de sa vie en prison pour avoir cru sérieusement à la réalisation imminente du Céleste royaume. En effet, son complot millénariste avait encouragé l'insurrection de la Calabre contre l'Antéchrist qui, dans les circonstances, avait l'aspect visible de l'Espagne. Rousseau avait assez de réalisme pour désespérer des grandes Babylones européennes, mais, en dépit des questions qu'il se posait par ailleurs, il jugeait ses propositions sur Genève, la Corse, la Pologne, suffisamment utiles pour se donner la peine de les rédiger. Fourier se présentait, dit-on, tous les midis sur la place du Palais-Royal pour recevoir de quelque riche mécène l'argent nécessaire pour réaliser les phalanstères de ses rêves.

Mais la volonté de créer un homme nouveau se heurte à des difficultés beaucoup plus grandes ici que dans l'ordre institutionnel ou instrumental. Rien n'est moins sûr que le progrès éthico-politique de l'humanité. Sur ce plan, rien n'est jamais définitivement acquis ; nulle civilisation n'est à l'abri des brusques retours de la barbarie. Les attitudes ne se sédimentent pas comme les connaissances et les objets de la technique. La courbe ascendante des " arts" et des sciences contraste avec la ligne brisée de l'histoire politique. La prétention progressiste affichée par les tenants de l'utopie vérité - de - demain n'atteint pas les profondeurs du cour humain. L'optimisme des utopistes - l'utopie est optimiste 
par définition - ne parvient pas à tourner en dérision la sagesse un peu triste des grands moralistes et des théoriciens réalistes de la politique dont on se défend mal en les appelant pessimistes. L'impitoyable lucidité des Machiavel, Hobbes, La Rochefoucauld, Kant, Schopenhauer, Pareto, Freud n'en continue pas moins de nous rappeler les limites du «progrès ». Thucydide, Tacite, Saint-Simon ont-ils peint une humanité tellement différente de la nôtre ? L'homme total de Marx n'est pas venu, mais "la grande terreur» et la bureaucratie du "gros animal » dont la dystopie d'Orwell et l'œuvre de Soljénitsyne décrivent le fonctionnement implacable. Les prophètes de la Redeemer Nation sont passés, et ceux que l'on disait «les meilleurs et les plus intelligents " ont semé la mort et la désolation avec une application perverse. L'éventualité du conflit est inhérente au monde des libertés. Rousseau l'a bien vu : l'ordre instable des passions obéit à une dialectique indépendante du progrès des lumières. L'amourpropre, les actes ténus de la conscience par lesquels chacun se préfere dans le secret de son cour et se sépare d'autrui, la sinistre triade de l'orgueil, de la haine et de la peur échappe au cumul indéfini des objets, des notions de même qu'aux innovations institutionnelles. Les mauvais usages de la liberté ont le même âge que l'exercice de la liberté elle-même. Rien ne nous permet d'affirmer qu'ailleurs ou dans un autre temps il puisse en être autrement. C'est pourquoi l'utopiste conséquent vise précisément à mettre hors jeu ce mystérieux pouvoir qui risque à tout moment de déjouer ses calculs d'un ordre et d'un bonheur sans failles. II n'a de cesse que chacun des gestes, chacun des mots de l'individu ne soit prédéterminé selon le plan total arrêté une fois pour toutes. Privé de liberté, soumis à un ordre estimé parfait et par suite définitif (bien qu’il soit toujours, en fait, l'œuvre d'un esprit fini), ce monde hypermoral est foncièrement antipolitique, antihistorique et finalement antimoral : il élimine dans le principe même l'événement, la surprise, la contingence, les conséquences non voulues des errances d'une liberté trop humaine entremêlée au cours capricieux de la Fortuna.

Le vice profond de la pensée utopique n'est pas de rêver d'une communauté fraternelle: cette noble visée ressortit 
légitimement à une "poétique de la volonté "; il est de croire qu'on puisse la réaliser immédiatement au niveau public de l'existence (ce qui implique logiquement la disparition du rapport hiérarchique et toujours à quelque degré violent de l'autorité). L'amour des hommes est un élan prophétique qui dépasse le moral et a fortiori la politique. Pour reprendre les expressions de P. Riccur : " la pédagogie violente du magistrat » est foncièrement irréductible à "l'éthique de la charité ", l'éthique sacrificielle du Sermon sur la montagne. Elle est dans l'existence humaine un moment nécessaire et indépassable. Certes, on peut concevoir que l'exigence hyperéthique d'une réciprocité non antagonistique entre les consciences vienne adoucir les rigueurs des nécessités politiques; cette requête ne peut pourtant pas aplatir la verticalité asymétrique du commandement et de la punition. Vouloir étendre les gratifications affectives caractéristiques des très petites communautés à de vastes ensembles sociaux, c'est acculer l'État à un forcing systématique dont il ne peut s'acquitter que par la terreur et la contrainte idéologique. Par sa finalité comme par ses moyens propres, la politique ne peut satisfaire cette demande qu'en pervertissant son essence et celle de la religion: la domination juste et raisonnable se dégrade en oppression fanatique, et la foi armée vire au salut forcé du grand Inquisiteur.

La nostalgie des origines ne peut être retenue à l'intérieur de l'organisation sociétaire moderne qu'à titre d'une exigence permanente d'une société plus juste, plus chaleureuse, plus heureuse, non comme la vision d'un état social parfait adopté comme un devis pour l'action immédiate. De la première fonction à la seconde, il y a toute la distance d'un " méliorisme » jamais satisfait de ses acquis à la synthèse trop vertueuse et trop impatiente de totalitarisme. Le rôle positif des utopies réside dans la tension prophétique qu'elles introduisent dans la vie sociale, relativisant sans cesse nos mœurs, nos pratiques, nos institutions, ouvrant toujours sur des possibilités nouvelles. Si la politique est l'art du possible et seulement du possible dans chaque situation, l'utopisme peut être l'aiguillon intérieur qui en fait l'art de tout le possible. Mais l'utopie de la communauté totale, parfaitement égalitaire 
et par conséquent sans autorité, soumet le corps social à une destination démesurée et la raidit dans la crispation autoritaire. Une telle idée ne peut être réalisée: elle ne peut être qu'espérée comme l'horizon le plus lointain de l'agir collectif. C'est indirectement, par la pression de l'espérance vécue sur l'action efficace, que l'utopisme édifie au lieu de s'évader dans la schizophrénie. Détachée de l'ordre prosaïque des moyens, la visée des fins s'égare dans le nirvâna d'un autre monde au lieu de lutter ici pour un monde autre.

Toute utopie inconsciente de sa démesure est grosse d'une tyrannie. L'agression est inhérente à la vie ; le «polythéisme des valeurs" dramatise tout ordre donné; il n'y a pas d'harmonie préétablie des intérêts et des passions : voilà pourquoi le politique sera toujours avec nous, mais ce n'est pas au grand Léviathan que revient la tâche eschatologique de la réconciliation totale, définitive. On peut déjà se réjouir s'il ne dévore pas ses propres sujets. À force de vouloir l'Harmonie totale, on institue la peur et l'hypocrisie générale, puis l'élimination systématique du suspect-traitre, enfin la guerre de tous contre tous. Le groupe se fortifie moralement dans cette saignée. La ferveur du «groupe en fusion» ne peut conserver son intensité fondatrice que par l'épuration permanente du Nous. D'autre part, toujours l'homme tend à reporter sur le Pouvoir l'espérance de l'établissement du règne des fins. Le monstre effrayant et fascinant de la Bible n'est-il pas, comme il est dit, la plus haute puissance sur la terre ? Or, en dépit du fait que l'idée d'une vaste communauté fondée sur l'amour soit manifestement une illusion, l'homme préfere s'abandonner à cette espérance impossible plutôt que de vivre sans espoir. Il faut que le centre brûlant de l'utopie corresponde à un tropisme fondamental de l'âme humaine pour que même dans la représentation rétrospective, les expressions symboliques d'un monde devenu enfin fraternel par la geste révolutionnaire apparaissent plus vraies que la dictature et la terreur. L'idée même des gains et du coût humain de la tentative paraît attentatoire à l'épopée de la libération.

Moins émotive, moins éruptive que le messianisme révolutionnaire, la construction utopique participe pourtant du même phantasme d'une societas perfecta, même si une telle 
prétention est davantage suggérée qu'affirmée. L'alliance jugée paradoxale de l'idéalisme religieux et de la violence politique dans les utopies écrites ou pratiquées depuis la Renaissance comme dans les millénarismes utopisants du Moyen Âge repose, comme l'a bien vu Mühlmann, sur une illusion, "l'illusion irénique». Il s'agit du "préjugé naif qui veut que la motivation religieuse soit aimante et pacifique de nature." En réalité, l'activisme politique sort directement de la mystique quiétiste elle-même. Saint-Just le disait déjà lorsqu'il définissait la Terreur comme l'impatience même de la Vertu. C'est l'excès de l'attente trop longtemps contenue qui explose en actes intolérants comme un arc trop bandé. Aussi, la volonté de puissance chez les utopistes présente-t-elle une qualité particulière qui ne saurait être confondue avec la simple passion du pouvoir : le Christ, Münzer, Robespierre, Marx, Gandhi ne sont pas de la même race que Gengis Khan, César, Napoléon, Pierre le Grand et Staline. Mais il n'est pas rare que les ambitieux, les arrivistes et les intrigants de basse espèce se faufilent dans les troupes de choc des militants-croyants quand le grand souffle s'atténue et confisquent le mouvement à leur profit.

Les valeurs les plus hautes qui composent l'horizon ultime de l'agir ne peuvent être objectivées qu'à travers une suite indéfinie d'approximations qui n'épuisent jamais l'absoluité de la visée axiologique. De ce point de vue, les institutions nouvelles trahissent autant qu'elles incarnent les nouvelles valorisations. À des fins abstraites absolues - Justice, Liberté, Fraternité, etc. - ne peuvent correspondre que des moyens extrêmes d'ailleurs intrinsèquement déficients en regard de l'aspiration utopique. D'où le caractère répétitif et perpétuellement récurrent de la dialectique Vertu-Terreur dont la Révolution française a fourni l'exemple éclatant.

Nul mieux que Hegel n'a saisi cette dialectique de l'objectivation et de l'aliénation non seulement dans toute sa généralité abstraite mais également dans le jeu des forces contradictoires de son époque. En réaction contre les «scissions» de l'homme moderne, il comprit la nécessité de la restauration d'un ordre communautaire, mais qui ne fût en rien l'involution antimoderne vers une imaginaire plénitude 
telle que la nostalgie de "la belle totalité grecque " pouvait en fournir la réverbération. L'antimanichéisme de la raison dialectique ne vise pas à l'élimination complète du négatif, mais à conduire à leur terme logique les tensions qui portent en elles la richesse intérieure de l'Esprit et les prémices d'un dépassement authentique. L'utopiste, au contraire, n'accepte pas la progression compliquée par «le mauvais côté » et les risques d'une synthèse différenciée. Il s'installe d'emblée dans la perfection d'une communauté surréglementée et retranchée.

Jusqu'à des temps relativement récents, l'humanité a vécu à l'intérieur de formations sociales réduites, simples, homogènes et fort peu ouvertes sur le monde extérieur assimilé au monde barbare. Si l'ethnologue s'enchante de la chatoyante diversité des peuples comme autant de variantes d'une humanité foncièrement une, les sociétés primitives vivent, elles, dans un régime d'ethnocentrisme aigu. Il est sûr que le sentiment d'appartenir à une petite communauté étroite, bien liée, unanime, procure à l'individu de très grandes satisfactions psychiques: la sécurité, la chaleur affective, le sentiment de puissance et de fierté. On peut même considérer avec Bergson que l'homme est fait pour vivre au sein de très petites sociétés; les vastes ensembles, hétérogènes, ouverts aux influences perturbatrices du dehors le jettent dans l'inconfort moral. De même que la psychanalyse nous assure de l'extraordinaire vivacité des expériences infantiles, la psychologie sociale et l'historiographie moderne affirment l'indéracinable attrait du phantasme archaique de la petite société close : la nostalgie de la tribu, tout comme la nostalgie du sein maternel, ne dépérit jamais tout à fait. Il est probable que toute mutation accélérée de la "communauté " en "société " moderne suscite par réaction «le retour du refoulé ".

Les utopies puisent leur énergie dans ces impérissables images. Tout projet utopique est dans son principe le projet d'une communauté amicale et fraternelle; toutes les différences internes du genre modulent sur cette visée fondamentale. Au-delà des techniques - miracles, au-delà de l'agencement formel des nouvelles institutions, le « noyau mythico-imaginatif " de l'utopie qui informe et vivifie la construction 
utopique réside dans cet élan enthousiaste vers une communauté parfaitement intégrée dont les membres sont intérieurement mus par l'attraction des mêmes valeurs.

Université de Montréal 